\title{
Validação psicométrica da Social Physique Anxiety Scale para brasileiros com amputação de membros
}

\section{Psychometric validation of Social Physique Anxiety Scale for Brazilian people with limb amputee}

(D) Roberto Gomes Ferreira Filho, (D)Angela Nogueira Neves ${ }^{2}$

${ }^{1}$ Escola de Educação Física do Exército - ESEFEX

\section{Correspondência}

Angela Nogueira Neves

E-mail: angelaneves.esefex@gmail.com

Submetido: 06 Março 2020 Aceito: 19 Novembro 2020

\section{Como citar}

Ferreira Filho RG, Neves AN. Validação psicométrica da Social Physique Anxiety Scale para brasileiros com amputação de membros. Acta Fisiatr. 2020;27(4):199-205.

DOI: 10.11606/issn.2317-0190.v27i4a178186

\section{RESUMO}

A ansiedade físico-social versa sobre a sensação de desconforto vivenciado quando há uma situação real ou imaginária, na qual parte ou todo o corpo é exposto ao julgamento de outra pessoa. Objetivo: Verificar a estrutura fatorial da versão brasileira da Social Physique Anxiety Scale em uma amostra de referência de pessoas amputadas. Métodos: Foi realizada análise fatorial confirmatória com os dados obtidos de uma amostra de 100 participantes, dos quais $99 \%$ eram homens, com idade média de 48,26 ( $\pm 18,26$; máx=82; mín= 15) anos e tempo médio de amputação de 76,36 ( $\pm 95,36$; máx= 504; mín=1) meses, para averiguação do melhor ajuste entre os modelos unidimensional e bidimensional. Foram calculados valores da confiabilidade de constructo e do teste de alpha de Conbrach para determinar evidências de confiabilidade. Para a validade de constructo, foram geradas evidências de validade convergente e discriminante. Evidências de validade convergente foram geradas pela variância média extraída (AVE) e pela análise da correlação com medida de constructo semelhante. Para a validade discriminante, foi verificado se havia a diferença teoricamente esperada entre dois grupos. Resultados: 0 modelo com melhor ajuste foi o unidimensional após a eliminação de quatro itens (RMSEA=0,074, $\chi 2 / g I=1,54, \mathrm{CFI}=1, \mathrm{NFI}=0,98, \mathrm{NNFI}=1, \mathrm{AGFI}=0,98, \mathrm{GFI}=0,99, \mathrm{AIC}=62,84$ ). As evidências de confiabilidade interna e discriminante foram satisfatoriamente geradas para o modelo de medida, havendo uma ressalva à validade convergente, com baixo valor de variância média extraída. Conclusão: A escala pode ser usada no Brasil para avaliar a ansiedade físico-social em pessoas amputadas.

Palavras-chaves: Imagem Corporal, Ansiedade, Psicometria, Estudo de Validação

\section{ABSTRACT}

Physical-social anxiety is about the sensation of discomfort experienced when there is a real or imagined situation, in which part or the whole body is exposed to the judgment of another person. Objective: To verify the factorial structure of the Brazilian version of the Social Physique Anxiety Scale in a reference sample of amputees. Methods: Confirmatory factor analysis was performed with data obtained from a sample of 100 participants, $99 \%$ of whom were men, with an average age of 48.26 ( \pm 18.26 ; $\max =82 ; \min =15)$ years, and time average amputation of 76.36 ( \pm 95.36 ; $\max =504 ; \min =1$ ) months, to determine the best fit between the one-dimensional and two-dimensional models. Construct reliability values and Conbrach's alpha test were calculated to determine evidence of reliability. For construct validity, evidence of convergent and discriminant validity was generated. Evidence of convergent validity was generated by the average extracted variance (AVE) and by the analysis of the correlation with a similar construct measure. For the discriminant validity, it was verified if there was a theoretically expected difference between two groups. Results: The model with the best fit was the one-dimensional model after the elimination of four items (RMSEA $=0.074, \chi 2 / d f=1.54, \mathrm{CFI}=1, \mathrm{NFI}=0.98$, NNFI= $1, \mathrm{AGFI}=0.98, \mathrm{GFI}=0.99, \mathrm{AIC}=62.84)$. Evidence of internal and discriminant reliability was satisfactorily generated for the measurement model, with a caveat to convergent validity, with a low value of the extracted average variance. Conclusion: The scale can be used in Brazil to assess physical and social anxiety in amputees.

Keywords: Body Image, Anxiety, Psychometrics, Validation Study 


\section{INTRODUÇÃO}

A ansiedade físico-social versa sobre a sensação de desconforto vivenciado quando há uma situação real ou imaginária, na qual parte ou todo o corpo é exposto ao julgamento de outra pessoa. Em outras palavras, é a tendência que uma pessoa pode ter de ficar apreensiva, quando acredita que o seu próprio corpo está sendo avaliado negativamente em uma situação social. ${ }^{1}$

A ansiedade físico-social pode ser considerada como uma experiência afetiva direcionada à própria identidade, sendo assim uma dimensão da imagem corporal. ${ }^{2}$ Esta é a representação mental do corpo, ${ }^{3}$ constituída em um mundo de relações e influenciada pelos aspectos fisiológicos, libidinais e o ambiente social. É dinâmica, singular, com aspectos conscientes e inconscientes. ${ }^{4} \mathrm{~A}$ imagem pode ser considerada uma estrutura e também um processo, ${ }^{5}$ uma vez que a representação mental do corpo forma a base de um senso de si e é continuamente atualizada de acordo com as experiências corporais. ${ }^{3,6}$ Neste sentido, ela pode ser entendida como um desenvolvimento dinâmico da representação mental self corporal. $^{7}$

Para pessoas com deficiência física adquirida, a reconfiguração do conceito de 'quem eu sou', que continua por muitos anos após a lesão, é um exemplo explícito do dinamismo da imagem corporal. As alterações que nela ocorrem após uma lesão neurológica - seja ela central ou periférica - geralmente são devidas à própria incapacidade, mas também são resultado de novas experiências corporais inerentes à atual condição. Eles variam de acordo com a história pessoal de cada pessoa e as circunstâncias em que a lesão ocorreu. ${ }^{8}$

A imagem corporal de uma pessoa com deficiência é moldada por percepções e atitudes em relação a um corpo 'não normal', e a pessoa deve lidar com a sua própria visão e a visão social de sua deficiência. ${ }^{9}$ Pessoas amputadas tendem a ser impactadas pelo olhar do outro, pois ele se dirige ao que falta, mais que ao sujeito em si. ${ }^{8}$ Considerando esses argumentos, as alterações na imagem corporal podem ser vistas como uma questão central no processo de ajuste enfrentado por indivíduos com uma deficiência adquirida. ${ }^{10}$

No processo de reabilitação, é necessário criar oportunidades de ressignificação da nova identidade corporal e acompanhar as alterações que ocorrem na imagem corporal, como em qualquer outro quadro neurológico. ${ }^{11} \mathrm{Um}$ ou mais instrumentos de medida psicometricamente ajustados são necessários para conduzir tal ação. ${ }^{12}$ Há tanto a possibilidade de haver uma ressignificação positiva quanto uma, aparentemente tranquila, estagnação traumática e por isso, o acompanhamento sistemático deve ser instituído nas unidades de reabilitação.

Avaliar o grau de desconforto, que esta população experimenta sob o olhar do outro, é importante, pois possibilita o acompanhamento do desenvolvimento da valorização pessoal. E para fazer isso, a ansiedade físico-social possui dois instrumentos de avaliação disponíveis: a Social Physique Anxiety Scale (SPAS) e a Social Appearance Anxiety Scale (SAAS). Porém, há diferença de foco entre esses instrumentos.

Enquanto a SPAS foca-se exclusivamente na ansiedade físico-social, a SAAS analisa a ansiedade social, considerando aspectos da aparência física. ${ }^{14} \mathrm{E}$ ainda, a SPAS é particularmente interessante para a área de educação física, esportes e de reabilitação cinesiomotora, pois foi criada considerando as experiências de desconforto vivenciadas durante a avaliação física em academias. ${ }^{1}$ Por estes dois motivos, a SPAS é alvo de estudo na presente pesquisa.

Originalmente, a SPAS tem uma estrutura unidimensional, com 12 itens, tendo sido validada em uma amostra de pessoas saudáveis, praticantes de exercício físico de ambos os sexos. ${ }^{1}$ Após a sua criação, a SPAS foi alvo de vários estudos que questionaram não somente a sua unidade fatorial, mas também a pertinência dos itens - com destaque do item 2. ${ }^{15-18}$

Um modelo bidimensional foi proposto ${ }^{16}$ e confirmado, ${ }^{17}$ assim como foi proposta nova redação ao item $2^{16}$ que esteve presente em subsequentes estudos psicométricos. ${ }^{17,18}$ Mas o estudo de Martin et al. ${ }^{19}$ gerou evidências mais robustas para confirmar o modelo unifatorial, com a redação original e todos os itens. A solução dessa abordagem mais robusta foi confirmada pelos estudos de Motl e Conroy. ${ }^{20,21}$

A SPAS já foi culturalmente adaptada e psicometricamente validada em outros países, como China, Japão, Coréia e Tailandia, ${ }^{22}$ Suécia, ${ }^{23}$ Portugal $^{24}$ e Brasil ${ }^{25-27}$ mantendo, em todos estes estudos, uma solução unifatorial, mas com redução de itens.

As amostras de referências utilizadas no Brasil para validar a SPAS foram de estudantes universitários, ${ }^{25}$ amostra combinada de estudantes, militares e pessoas da comunidade ${ }^{26}$ e de pessoas praticantes de exercício físico, oriundas de clubes e universidades. ${ }^{27}$ Não houve ainda nenhum estudo com pessoas amputadas, público alvo do presente trabalho. Fazer o estudo psicométrico para esta população suprirá esta lacuna metodológica, e também oferecerá um instrumento adequado para ser incorporado aos protocolos de avaliação e acompanhamento do processo de reabilitação.

A Psicometria é uma área da estatística destinada a gerar evidências confirmatórias, que o instrumento de medida realmente avalia o que se propôs a medir - validade - e que o faz sem erros sistemáticos - confiabilidade. ${ }^{28}$

A validade pode ser principalmente de três tipos: conteúdo, critério e constructo. A validade de constructo é a forma mais fundamental e consiste em verificar se a escala constitui uma representação legítima e adequada do constructo teórico, ou seja, verifica se os dados coletados confirmam a previsão teórica feita pela medida. É considerada um tipo de validade mais sofisticada e difícil de estabelecer. ${ }^{29}$

A confiabilidade se refere tanto à estabilidade quanto à acurácia. Para escalas de medida, é relevante um tipo de confiabilidade relacionada à acurácia, a consistência interna. ${ }^{30}$

É importante afirmar que as evidências de validade e confiabilidade não pertencem ao instrumento per se, mas se associam ao desempenho do instrumento em amostras de referências, atestando a adequação do instrumento para avaliação das mesmas. ${ }^{29}$

\section{OBJETIVOS}

Frente à ausência de estudos com amputados e a pertinência de ter um instrumento capaz de medir corretamente os traços de ansiedade físico-social para este público, o objetivo desta pesquisa é verificar a estrutura fatorial 
da versão brasileira da Social Physique Anxiety Scale $e^{25}$ em uma amostra de referência de pessoas amputadas, gerando evidências de consistência interna e validade de construto.

\section{MÉTODO}

Este trabalho teve delineamento de estudo metodológico. ${ }^{31}$ Teve como público-alvo pessoas com amputação, com uma amostragem não probabilística por julgamento. Como critério de inclusão adotamos: pessoas de ambos os sexos com 18 anos ou mais; com amputação de membro superior ou inferior ou ambas; sendo a amputação de causa traumática ou circulatória.

Como critério de exclusão adotamos: protetização ocorrida há mais de cinco anos; presença de má formação congênita que tenha causado ausência de membro. Aqueles que responderam a todos os questionários e não assinaram ao Termo de Consentimento Livre e Esclarecido (TCLE) tiveram os dados excluídos da análise.

O tamanho amostral foi calculado se usando a regra de bolso para análise fatorial confirmatória, ${ }^{29}$ que determina ser necessário haver, pelo menos, cinco respondentes para cada item da escala, acrescendo $20 \%$ a mais para eventuais perdas de dados. Dessa forma: tamanho amostral = $(5 \times 12$ itens da SPAS) $+20 \%=75$ respondentes foi considerado tamanho amostral mínimo para esta pesquisa.

\section{Instrumentos}

- Social Physique Anxiety Scale (SPAS): ${ }^{1}$ foi criada para avaliar o grau de ansiedade experimentado pela pessoa, quando outros estão efetivamente observando ou avaliando o seu corpo, ou quando há a expectativa de que isso ocorra. A versão brasileira da SPAS $^{25}$ apresentou estrutura unifatorial reduzida para homens e mulheres com 9 e 10 itens, respectivamente. Para o presente estudo, trabalhou-se com os 12 itens originais traduzidos, com o item 2 invertido, ${ }^{16}$ sendo reversos os itens 1 , 2, 5, 8 e 11 .

As opções de respostas estão dispostas em uma escala tipo Likert de cinco pontos ( $1=$ nada parecido comigo a $5=$ tudo a ver comigo). O escore final da escala é dado pela média de todos os itens. Quanto maior o escore, maior é o grau de ansiedade físico-social.

- Amputee Body Image Scale (ABIS): ${ }^{32}$ foi criada para avaliar se a amputação provocou alterações na imagem corporal, tendo como referencial teórico os critérios diagnósticos para dismorfia corporal do DSM-IV, e como isso afeta a experiência corporal da pessoa com amputação. A versão Brasileira da $\mathrm{ABIS}^{33}$ teve um bom ajuste para uma amostra de referência de amputados, com estrutura fatorial distinta da original e com redução de itens.

Os fatores são: (1) preocupação com a aparência: itens 1, 2, 6, 8, 10, 14 e 18; (2) Prótese e coto, itens 5, 12, e 20; (3) Função e deficiência, itens 4, 9, 11 e 15 (numerações referentes à escala original).

As respostas estão dispostas numa escala tipo Likert de 5 pontos ( $1=$ nunca, $5=$ sempre). Altos escores indicam um nível mais elevado de alterações na imagem corporal.

\section{Procedimentos}

A coleta de dados foi realizada em dois locais distintos, a saber: o posto de perícia do INSS e no Centro de Reabilitação Lucy Montoro (Unidade Campinas). No INSS, os participantes foram abordados na fila de espera da perícia e no Centro de Reabilitação, na sala de espera das terapias. Em ambos os lugares, foi explicado o objetivo da pesquisa $e$ os procedimentos para a participação, esclarecendo que a mesma seria de forma voluntária.

Aos indivíduos que aceitaram participar, foi lhes entregue o TCLE em uma prancheta. Após leitura e assinatura do TCLE, foi entregue a escala para preenchimento.

Foi utilizado nesta pesquisa, o método estatístico de análise fatorial confirmatória, por apresentar mais consistência e robustez em relação à sua alternativa, a análise fatorial exploratória. ${ }^{34}$ Foram utilizados os softwares SPSS ${ }^{\circledR 22}$ e LISREL ${ }^{\circledR}$ 8.51 além do módulo Prelis $^{\text {Tm } 2}$ do sistema LISREL $^{\circledR}$ para preparação dos dados. Foram investigados nessa pesquisa, dois modelos estruturais, anteriormente determinados para a SPAS: o modelo original, ${ }^{1}$ com dois fatores e o modelo bifatorial. ${ }^{16}$

\section{Critérios para análise e ajuste do modelo estrutural}

A investigação de validade foi feita sob o modelo de análise proposto por Anastasi e Urbina, ${ }^{35}$ Marôco, $^{36}$ Hair et al. ${ }^{29} \mathrm{E}$ especificamente para investigação da validade discriminante, seguimos as diretivas de Paquali. ${ }^{30}$ Aqui, a validade de construto é entendida como tendo três subcomponentes: validade fatorial; validade convergente e validade discriminante.

De acordo com essas referências, a validade fatorial é investigada por meio da verificação do próprio modelo de escala, avaliando o ajuste do modelo por meio dos índices de ajuste, ${ }^{36}$ os quais adotamos: (1) Raiz do Erro Quadrático Médio de Aproximação (RMSEA): foi adequado valor abaixo de 0,08,29 (2) Qui-quadrado Normado ( $\chi 2$ /graus de liberdade): foi considerado como ideal, valores entre dois e três, sendo aceitável valor abaixo de cinco; ${ }^{37}$ (3) Índice de Ajuste Normado (NFI) e Índice de ajuste de critério (CFI): foi considerado adequado valor igual ou superior a 0,$9 ;^{29}$ (4) Critério de Informação Akaike (AIC): valores mais próximos de zero indicam maior parcimônia estatística. ${ }^{29}$

O ajuste do modelo estrutural deu-se por consecutivos ajustes apoiados pelo sistema LISREL ${ }^{\circledR}$ que forneceu as cargas fatoriais, os erros associados aos itens e os índices de modificações, possibilitando eliminar e/ou reter itens. Para cada modificação no modelo foi feita uma nova estimação, buscando sempre atingir limites preconizados de qualidade de ajuste.

Quanto às técnicas de estimação dos modelos, utilizamos o método de Mínimos Quadrados Não-Normados (ULS) ${ }^{38}$ e foi adotada deleção listwise para os dados faltantes. Para verificação da multinormalidade, foi adotado como parâmetros curtose (Ku) e assimetria (SK), sendo indicadores de multinormalidade $\mathrm{Ku}<|3|$ e $\mathrm{Sk}<|10| .{ }^{29}$

\section{Critérios para análise e ajuste do modelo mensuração}

Após o ajuste do modelo estrutural, avaliamos o ajuste no modelo de mensuração, para determinar a confiabilidade interna e a os outros dois subcompomentes da validade da escala. Seguindo o protocolo escolhido, para a determinação das evidências de confiabilidade, calculamos os valores da 
confiabilidade de constructo (quadrado da soma das cargas fatoriais/(quadrado da soma das cargas fatoriais + soma dos erros)) e do teste Alpha $(\alpha)$ de Conbrach. Ambos geram evidência de um tipo específico de confiabilidade, a consistência interna. Para ambos, valores maiores ou iguais a 0,70 são adequados. ${ }^{39}$

Ainda de acordo com as referências metodológicas adotadas, geramos evidências de validade discriminante e convergente. Para este último, calculamos a variância média extraída (AVE; soma das cargas fatoriais ao quadrado / (soma das cargas fatoriais ao quadrado + soma dos erros)), que indica a quantidade total de variância dos indicadores explicada pela variável latente. Valores acima de 0,50 foram considerados adequados. ${ }^{40}$

Também adotamos uma segunda abordagem, na qual se atesta a validade convergente a partir da correlação de outra medida de constructo semelhante. Fizemos uma análise de correlação com os escores dos fatores da ABIS. Correlações significantes indicam convergência entre as medidas. ${ }^{30} \mathrm{O}$ teste de normalidade de Kolmogorov-Smirnov para os escores das medidas indicou não aderência à normalidade, e assim, foi utilizado o teste de correlação de Spearman.

$\mathrm{Na}$ impossibilidade de usar o critério de Fornell e Larcker ${ }^{40}$ para o modelo unifatorial, para a validade discriminante, comparou-se dois grupos que, nos quais teoricamente, deveria se esperar manifestações distintas da ansiedade físico-social: pessoas fisicamente ativas e sedentárias. ${ }^{25}$ Diferenças significantes - no caso, no teste $U$ de Mann-Whitney - indicam que a escala é sensível para discriminar grupos. ${ }^{30}$ Estas análises univariadas foram feitas no SPSS, versão $22 \mathrm{e}$, para todas as análises, foi considerado $\alpha=0,05$.

\section{Aspectos éticos}

Este estudo fez parte de um projeto maior que, atendeu às Portarias CNS 466/12 e CNS 510/16, tendo seguido também princípios éticos contidos na Declaração de Helsinki, aprovado no comitê de ética em pesquisa da Universidade Estadual de Campinas (UNICAMP), CAAE: 19330713.8.0000.5404.

\section{RESULTADOS}

A amostra do estudo foi composta por 100 participantes, dos quais $99 \%$ eram homens, com idade média de 48,26 ( \pm 18,26; máx=82; mín=15) anos, e tempo médio de amputação de 76,36 ( $\pm 95,36 ;$ máx $=504 ;$ mín= 1) meses. Quanto à localização da amputação, $24 \%$ eram nos membros superiores, $74 \%$ nos membros inferiores e $2 \%$ em ambos os membros. Por fim, $66 \%$ faziam atividade física regularmente e $34 \%$ se declararam sedentários.

Os resultados descritivos iniciais indicaram uma tendência a escores mais inferiores nos itens da SPAS, evidenciando uma possível manifestação menos intensa de ansiedade físico-social nessa amostra. Também evidência que todos os itens alcançaram valores máximos e mínimos de repostas, indicando que as variáveis observáveis puderam avaliar diferentes de manifestações do constructo (Tabela 1).

Os indicadores de assimetria e curtose das respostas a cada item indicam aderência à multinormalidade, permitindo dar continuidade ao plano de análise original.
Tabela 1. Dados descritivos e indicadores de multinormalidade dos itens da SPAS

\begin{tabular}{lccccc}
\hline Item & $\begin{array}{c}\text { Valor } \\
\text { máx. }\end{array}$ & $\begin{array}{c}\text { Valor } \\
\text { mín. }\end{array}$ & $\begin{array}{c}\text { Média } \pm \\
\text { DP }\end{array}$ & Assimetria & Kurtose \\
\hline $1^{*}$ & 5 & 1 & $2,87 \pm 1,35$ & 0,18 & $-0,87$ \\
$2^{*}$ & 5 & 1 & $1,69 \pm 0,92$ & 0,82 & $-0,39$ \\
3 & 5 & 1 & $2,20 \pm 1,34$ & 0,45 & $-1,02$ \\
4 & 5 & 1 & $1,90 \pm 1,10$ & 0,65 & $-0,68$ \\
$5^{*}$ & 5 & 1 & $3,01 \pm 1,40$ & 0,09 & $-0,98$ \\
6 & 5 & 1 & $2,14 \pm 1,02$ & 0,36 & $-0,67$ \\
7 & 5 & 1 & $2,12 \pm 1,15$ & 0,43 & $-0,76$ \\
$8^{*}$ & 5 & 1 & $3,20 \pm 1,43$ & 0,04 & $-1,06$ \\
9 & 5 & 1 & $2,19 \pm 1,20$ & 0,36 & $-0,85$ \\
10 & 5 & 1 & $2,35 \pm 1,33$ & 0,41 & $-0,90$ \\
11 & 5 & 1 & $2,37 \pm 1,35$ & 0,04 & $-0,54$ \\
12 & 5 & 1 & $2,27 \pm 1,31$ & 0,47 & $-0,89$ \\
\hline
\end{tabular}

* itens com escore reverso; DP = desvio-padrão

\section{Ajuste do modelo estrutural}

A primeira estimação do modelo 1 , unifatorial, obteve um ajuste razoável (RMSEA $=0,126, \chi 2 / g I=2,57, C F I=0,44, N F I=$ $0,87, \mathrm{Al}=186,95)$. Porém os itens $1(\lambda=0,38), 3(\lambda=0,47), 5(\lambda=$ $0,27), 8(\lambda=0,05)$ e $11(\lambda=0,10)$ tiveram carga fatorial mais baixa. 0 melhor ajuste do modelo 1 ocorreu após eliminarmos os itens $1,5,8$ e 11 e aceitarmos o item $3(\lambda=0,48)$ com sua carga fatorial pouco abaixo do tolerável (RMSEA $=0,074, \chi 2 / g \mid=$ $1,54, \mathrm{CFI}=1, \mathrm{NFI}=0,98, \mathrm{AIC}=62,84)$. As cargas fatoriais variaram entre $\lambda=0,48$ a $\lambda=0,77$ (Figura 1).

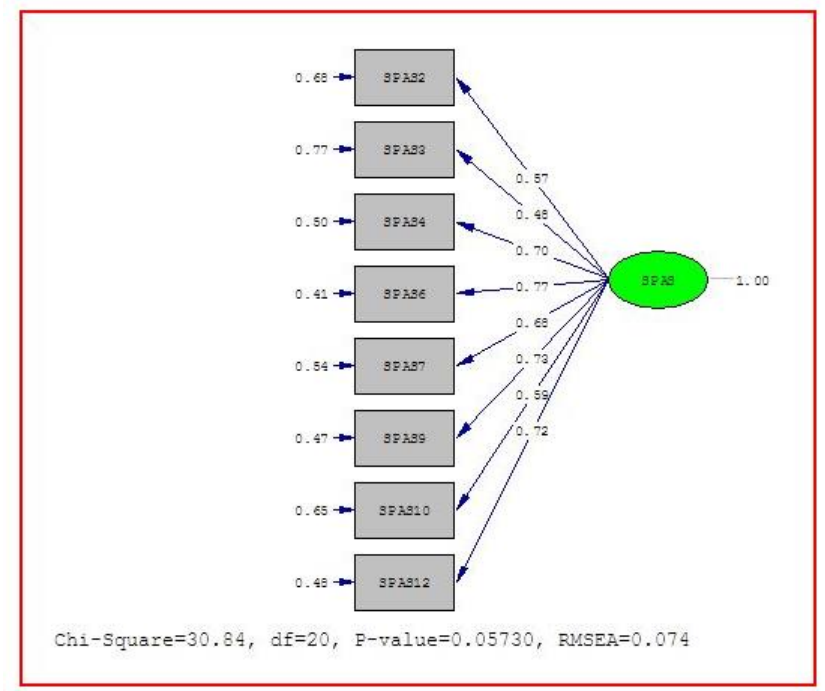

Figura 1. Modelo unifatorial ajustado da SPAS para uma amostra de Brasileiros amputados

O modelo 2, bifatorial, apresentou ajuste quase satisfatório na primeira estimativa (RMSEA $=0,084, \chi 2 / \mathrm{gl}=1,69, \mathrm{CFI}=0,998$, $\mathrm{NFI}=0,92, \mathrm{AIC}=139,75)$. Dos três itens com baixa carga fatorial, apenas o item $8(\lambda=0,22)$ pode ser eliminado do modelo, pois os itens $3(\lambda=0,47)$ e $11(\lambda=0,35)$ tiveram que ser mantidos para manutenção do fator Conforto Físico.

Todavia, se assim precedêssemos, a qualidade da medida seria comprometida - pois se manteria um fator fraco no modelo - e então, a solução foi a eliminação do fator. Com essa ação obtivemos a mesma estrutura fatorial do modelo 
unifatorial, ficando evidente ser ele a estrutura mais parcimoniosa para estes dados (Figura 1).

Evidências de confiabilidade interna, validade convergente e discriminante da SPAS

Em relação à confiabilidade interna, foram geradas evidências satisfatórias, sendo $\alpha=0,82$ e $C C=0,86$. Quanto à validade convergente, para o único fator ansiedade físico-social o valor de AVE foi inferior ao aceitável $(A V E=0,44)$. Mas alguma evidência de validade convergente foi gerada com a correlação significativa dos escores dos fatores da ABIS com o escore da SPAS (Tabela 2).

Tabela 2. Correlações dos Escores a SPAS e fatores da ABIS

\begin{tabular}{|c|c|c|c|c|}
\hline & 1 & 2 & 3 & 4 \\
\hline 1. SPAS & - & & & \\
\hline $\begin{array}{l}\text { 2. Preocupação com } \\
\text { corpo - ABIS }\end{array}$ & $0,543 * *$ & - & & \\
\hline 3. Prótese e Coto - ABIS & $0,431 * *$ & $0,614 * *$ & - & \\
\hline $\begin{array}{l}\text { 4. Função e Deficiência - } \\
\text { ABIS }\end{array}$ & $0,559 * *$ & $0,747^{* *}$ & $0,537 * *$ & - \\
\hline
\end{tabular}

** correlações significantes à 0,01

Em relação à validade discriminante, o teste $U$ de Mann Whitney foi significante, $U=834, p=0,04, r=0,21$, indicando que o grupo sedentário (rank médio $=54,86$ ) tinha maiores escores de ansiedade físico-social que o grupo fisicamente ativo (rank médio $=42,03$ ).

\section{DISCUSSÃO}

Este trabalho se propôs a verificar a estrutura fatorial da SPAS, ${ }^{25}$ gerando evidências de confiabilidade interna e validade de construto, em uma amostra constituída de pessoas amputadas.

O modelo unifatorial aqui encontrado já se mostrou anteriormente aderente no Brasil, ${ }^{23-25,27}$ e em outros países. ${ }^{22}$ Todavia não há uma estabilidade em relação aos itens: há variação tanto na quantidade de itens e em relação à quais itens são retidos nos modelos. Isso indica que a manifestação da ansiedade físico-social tem distinções entre culturas e amostras de referências.

Tal fato deve deixar o pesquisador atento à escolha da versão desse instrumento, vindo sempre a buscar aquela testada em um público-alvo semelhante àquele que ele busca avaliar. Por outro lado, a estrutura unifatorial indica aderência à teoria que fundamenta a escala e, adiciona mais uma evidência de que a estrutura bifatorial deve-se mais à um efeito de item wording - pois em um fator apenas se aglomeram os itens reversos - que teórica. ${ }^{19-21}$

Quanto à consistência interna, a escala mostrou-se capaz de medir a ansiedade físico-social de maneira confiável, apresentando valores satisfatórios. Valores bem aproximados aos aqui gerados foram encontrados em outras versões brasileiras, Campana et al. ${ }^{24}$ encontraram $\alpha=0,82$ e $\mathrm{CC}=0,81$; Neves et al. ${ }^{26}$ obtiveram na escala feminina $\alpha=0,87$ e $C C=0,86$ e na masculina $\alpha=0,81$ e $C C=0,85$; Souza e Fernandes ${ }^{27}$ realizaram apenas o teste de alpha de Cronbach, encontrando $\alpha=0,82$. Os nossos achados e os anteriores são levemente inferiores aos encontrado no estudo de desenvolvimento da SPAS1, $\alpha=0,90$, mas reforçamos, ainda adequados.

Quanto ao valor inferior da AVE encontrado, que não gerou uma evidência satisfatória de validade convergente, há uma interpretação que pode ser feita em relação à manifestação da ansiedade físico-social no grupo de referência, ora analisado. Podemos considerar que aparência e função do corpo interferem profundamente na maneira como interagimos no mundo social e os outros reagem a nós. ${ }^{41}$

A imagem corporal da pessoa com deficiência é modelada nesse contexto de interações e reações a partir de um corpo diferente da norma, que exerce sua função de locomoção parcialmente diferente - especialmente quando não ocorreu a protetização. ${ }^{33}$ Ademais, há modificações na imagem corporal decorrentes de reajuste da representação mental do corpo após a lesão nervosa decorrente da amputação - seja ela de causa traumática ou circulatória. ${ }^{42}$

Esta população se depara então com o desafio de recuperar as funções do corpo e aceitar perdas e alterações da aparência inveitáveis. ${ }^{33,43}$ Dado essa dinâmica no desenvolvimento da imagem corporal característico da pessoa com deficiência, entendemos que o baixo valor de AVE encontrado pode ser um indicativo de ausência de variáveis observáveis para mensurar especificamente a experiência da amputação e ansiedade físico-social, pois os indicadores são pertinentes, mas não estão representando tão eficazmente o constructo.

Todavia, foi encontrada a correlação significante com outra medida de avaliação da imagem corporal, psicometricamente apropriada para a amostra de referência, o que indica que a SPAS avalia traços da imagem corporal. A diferença significante entre pessoas ativas e sedentárias comprova que a escala discrimina grupos nos quais seriam esperadas manifestações distintas da ansiedade físico-social.

Observa-se que essa diferença é reconhecida na literatura, porém não há apontamento de qual dos dois grupos sedentários ou fisicamente ativos - apresenta maior manifestação da ansiedade físico-social. Por um lado, assumese que indivíduos com altos níveis de ansiedade física social são mais propensos a evitar a prática de exercícios em ambientes públicos, ou em situações onde seu corpo possa ser revelado, como em uma piscina pública. ${ }^{44}$

De outra forma, pessoas mais ativas são menos propensas à ansiedade físico-social, uma vez que o exercício ajuda a alcançar um corpo mais próximo dos padrões sociais, diminuindo assim a expectativa de uma reação negativa. ${ }^{45,46}$

Estudos experimentais indicam a diminuição da ansiedade físico-social após a prática de um programa de exercícios. ${ }^{47,48}$ Quando voltados especificamente às pessoas com deficiência, os estudos sobre esta evidência focam-se apenas em atletas de sucesso. ${ }^{49,50}$

Ao gerar resultados demonstrando que pessoas fisicamente ativas, não atletas, também apresentam menor intensidade da ansiedade físico-social, este estudo fornece novas informações acerca da temática, complementando a literatura pré-existente e enfatizando a necessidade de realização de atividades de condicionamento físico com esse publico.

Apesar de disponibilizar um novo instrumento para avaliação da pessoa com deficiência, o presente estudo tem limitações. Sua amostra foi constituída, em sua grande maioria, por homens, o que implica na restrição do tipo de população 
na qual a escala pode ser aplicada - preferencialmente para o público masculino. Ademais, o tamanho amostral foi bem próximo ao limite mínimo recomendado e novos estudos, com maiores amostras, podem ser interessantes para o contínuo refinamento da medida.

Nesse sentido, recomendamos também que outros estudos, com outras abordagens psicométricas sejam conduzidos, inclusive com a proposição de uma medida revista, com itens propostos especificamente para este público aqui analisado.

\section{CONCLUSÃO}

O trabalho alcançou o objetivo de verificar a estrutura fatorial da Versão Brasileira da SPAS, ${ }^{25}$ através de validação psicométrica, tendo como referência uma amostra de pessoas amputadas. Após análise e discussão dos dados constatou-se que retirando os itens $1,5,8$ e 11, a escala se comporta de modo satisfatório, mostrando-se capaz de medir o constructo ansiedade físico-social de maneira confiável e consistente, apresentando evidência mais robusta de consistência interna e validade discriminante e evidência menos forte, mas ainda pertinente, de validade convergente.

Quanto à aplicação na população, aconselha-se a utilizá-la somente em homens, tendo em vista o baixo número de mulheres na amostra. Assim, o instrumento pode ser utilizado em unidades de reabilitação para realizar o acompanhamento, especialmente, dos homens que tiveram amputação de membros superiores ou inferiores nele cadastrados. Desta maneira, este estudo viabiliza para os profissionais da área da Saúde uma ferramenta capaz de medir a ansiedade físico-social e possibilita uma melhor intervenção e planejamento do tratamento no País.

\section{REFERÊNCIAS}

1. Hart EA, Leary MR, Rejesky WJ. The measurement of Social Physique Anxiety. J Sport Exerc Psychol. 1989;11(1):94-104.

Doi: https://doi.org/10.1123/jsep.11.1.94

2. Campana ANNB, Tavares MCGCF. avaliação da imagem corporal: instrumentos e diretrizes para pesquisa. São Paulo: Phorte; 2009.

3. Schilder P. The image and appearance of the human body. New York: International Universities Press; 1980.

4. Tavares MCGCF. Imagem corporal: conceito e desenvolvimento. São Paulo: Barueri; 2003.

5. Shontz FC. perceptual and cognitive aspects of body experience. New York: Academic Press; 1969.

6. Shontz FC. Body image and physical disability. In: Cash TF, Pruzinsky T. Body images: development, deviance and change. New York: Guilford; 1990. p. 149-68.

7. Krueger DW. Psychodynamic perspectives on body image. In: Cash TF, Pruzinsky T. Body image: a handbook of theory, research and clinical practice. New York: Guilford; 2004. p. 30-7.

8. Murray CD, Fox J. Body image and prosthesis satisfaction in the lower limb amputee. Disabil Rehabil. 2002;24(17):925-31.

Doi:
9. Neves AN, Lorey GA, Campana MB, Ferreira L, Silva D. Factor structure, validity and internal consistency of the Body Appreciation Scale for Brazilian physically active spinal cord injured men. Acta Fisiátr. 2015;22(2):77-82. Doi: https://doi.org/10.5935/0104-7795.20150020

10. Harcourt D, Rumsey N. Body Image and biomedical interventions for disfiguring conditions. In: Cash T, Smolak L. Body image: a handbook of science, practice and prevention. $2^{\text {nd }}$ ed. New York: Guilford; 2011. p. 404-14.

11. Tavares MCGCF, Campana ANNB. Imagem corporal em pessoas com esclerose múltipla ativas e sedentárias. Acta Fisiátr. 2012;19(1):26-31. Doi: https://doi.org/10.5935/0104-7795.20120006

12. McDowell I, Newell C. Measuring health: a guide for rating scales and questionnaires. Oxford: Oxford Press; 1996.

13. Cyrulnik, B. Os patinhos feios. São Paulo: Martins Fontes; 2004.

14. Hart TA, Flora DB, Palyo SA, Fresco DM, Holle C, Heimberg RG. Development and examination of the social appearance anxiety scale. Assessment. 2008;15(1):48-59. Doi: https://doi.org/10.1177/1073191107306673

15. Eklund RC, Kelley B, Wilson P. The Social Physique Anxiety Scale: Men, women, and the effects of modifying Item 2. J Sport Exerc Psychol.1997;19(2):188-96.

16. Eklund RC, Mack D, Hart E. Factorial validity of the social physique anxiety scale for females. J Sport Exerc Psychol. 1996;18(3):281-95.

https://doi.org/10.1123/isep.18.3.281

17. McAuley E, Burman G. The Social Physique Anxiety Scale: construct validity in adolescent females. Med Sci Sports Exerc. 1993;25(9):1049-53.

18. Petrie TA, Diehl N, Rogers RL, Johnson C. The social physique anxiety scale: reliability and construct validity. J Sport Exerc Psychol. 1996;18(4):420-25. Doi: https://doi.org/10.1123/jsep.18.4.420

19. Martin JJ, Kliber A, Kulinna PH, Fahlman M. Social physique anxiety and muscularity and appearance cognitions in college men. Sex Roles. 2006;55(1):151-8.

20. Motl RW, Conroy DE. Validity and factorial invariance of the social physique anxiety scale. Med Sci Sports Exerc. 2000;32(5):1007-17. https://doi.org/10.1097/00005768-200005000-00020

21. Motl RW, Conroy DE. The social physique anxiety scale: cross validation, factorial invariance, and latent mean structure. Meas Phys Educ Exerc Sci. 2001;5(2):81-95. Doi: https://doi.org/10.1207/S15327841MPEE0502 2

22. Isogay $\mathrm{H}$, Brewer BW, Cornelius $\mathrm{AE}$, Komiya $\mathrm{S}$, Tokunaga $\mathrm{M}$, Tokushima S. Cross-cultural validation of the social physique anxiety scale. Int J Sport Psychol. 2001;32(1):7687.

23. Lindwall M. Factorial Validity and Invariance Testing of the Swedish Social Physique Anxiety Scale: Arguments for Gender-Specific Scales. J Sport Exerc Psychol. 2004;26(1):492-9.

https://doi.org/10.1123/jsep.26.3.492

24. Malheiro AS, Gouveia MJ. Ansiedade física social e comportamentos alimentares de risco em contexto desportivo. Anal Psicol. 2001;19(1):143-55. 
25. Campana ANNB, Tavares MCGCF, Silva D. Tradução, adaptação cultural e validação da Social Physique Anxiety Scale, para homens, para a língua Portuguesa no Brasil. In: VIII Congresso Iberoamericano da Avaliação Psicológica/XV Conferencia Internacional de Avaliação Psicológica; 2011 Jul 25-7; Lisboa. Atas do VIII Congresso Iberoamericano da Avaliação Psicológica/XV Conferencia Internacional de Avaliação Psicológica. Lisboa: Sociedade Portuguesa de Psicologia; 2011. p. 836-49.

26. Neves AN, Neves AB, Zanetti MC, Brandão MRF, Ferreira L. Validação psicométrica da Social Physique Anxiety Scale no Brasil. Rev Iberoam Psicol Ejerc Deport. 2018;13(2):193-202.

27. Souza V, Fernandes S. Adaptação da Social PhysiqueAnxiety Scale (SPAS) ao contexto brasileiro. Cien Cogn. 2009;14(3):16-23.

28. Malhotra NK. Pesquisa de Marketing: uma orientação aplicada. 4 ed. Porto Alegre: Bookman; 2004.

29. Hair Jr JF, Black WC, Babin BJ, Anderson RE, Tatham RL. Análise multivariada de dados. 6 ed. Porto Alegre: Bookman; 2009.

30. Pasquali L. Instrumentação psicológica: fundamentos e práticas. Porto Alegre: Artmed; 2010.

31. Mauch JE, Birch JW. Guide to the successful thesis and dissertation: a handbook for students and faculty. $4^{\text {th }}$ ed. New York: CRC Press; 1998.

32. Breakey JW. The lower limb amputee. J Prosthet Orthot. 1997;9(2):58-69.

33. Ferreira L, Meregui AGM, Mainenti MRM, Vigário PS, Neves AN. Brazilian Portuguese version of the Amputee Body Image Scale: cultural adaptation and a psychometric analysis. Percept Mot Skills. 2018;125(3):507-524. Doi: https://doi.org/10.1177/0031512518767755

34. Brown TA. Confirmatory factor analysis for applied research. New York: Guilford; 2006.

35. Anastasi A, Urbina S. Testagem psicológica. Porto Alegre: Artes Médicas; 2000.

36. Marôco J. Análise de equações estruturais. Pêro Pinheiro: Report Number; 2014.

37. Maruyama GM. Basics of estructural equation modeling. Thousand Oaks: SAGE; $1998 . \quad$ Doi: http://dx.doi.org/10.4135/9781483345109

38. Hoyle RH. Structural equation modeling - concepts, issues, and applications. Thousand Oaks: SAGE; 1995.

39. Hair Jr JF, Anderson RE, Tatham RL, Black WC. Análise multivariada de dados. 5 ed. Porto Alegre: Bookman; 2005.

40. Fornell C, Larcker D. Structural equation models with unobservable variables and measurement error. J Mark Res. 1981;18(1):39-50. Doi:
41. Cash TF. The body image workbook: An 8-step program for learning to like your looks. Oakland: New Harbinger; 1997.

42. Crerand CE, Magee L. Amputations and prosthetic devices. In: Cash TF. Encyclopedia of body image and human appearance. London: Elsevier; 2012. p.1-7.

43. Tavares MCGCF. Imagem Corporal e qualidade de vida. In: Gonçalves A, Vilarta R. Qualidade de vida e atividade física: explorando teorias e práticas. São Paulo: Manole; 2004. p.79-102.

44. Davison TE. Body Imagem in social contexts. In: Cash TF. Encyclopedia of body image and human appearance. London: Elsevier; 2012. p. 243-49.

45. Crocker P, Sabiston C, Forrestor S, Kowalski N, Kowalski K, McDonough $\mathrm{M}$. Predicting change in physical activity, dietary restraint, and physique anxiety in adolescent girls: examining covariance in physical self-perceptions. Can J Public Health. 2003;94(5):332-7. Doi: https://doi.org/10.1007/BF03403555

46. Brewer BW, Diehl NS, Cornelius AE, Joshua MD, Van Raalte JL. Exercising caution: social physique anxiety and protective self-presentational behaviour. J Sci Med Sport. 2004;7(1):47-55. Doi: https://doi.org/10.1016/s14402440(04)80043-4

47. Bartlewski PP, Van Raalte JL, Brewer BW. Effects of aerobic exercise on the social physique anxiety and body esteem of female college students. Women Sport Phys Act J. 1996;5(2):49-62. Doi: https://doi.org/10.1123/wspaj.5.2.49

48. Williams PA, Cash TF. Effects of a circuit weight training program on the body images of college students. Int J Eat Disord. 2001;30(1):75-82. Doi: https://doi.org/10.1002/eat.1056

49. Demirel H. Social Appearance Anxiety and Rosenberg SelfEsteem Scores in Young Physical Disabled Athletes. Univers J Educ Res. 2019;7(3):664-7. Doi: https://doi.org/10.13189/UJER.2019.070304

50. Piletic C. Athletes with lower limb paralysis' motives for competing in powerlifting [Dissertation]. Denton: Texas Woman's University; 1998. 